Duazary / Vol. 16, No. 2 - 2019 / 189 - 190

Doi: https://doi.org/10.21676/2389783X.2758

\title{
EDITORIAL 2
}

\section{Entrevistas en los procesos de selección de estudiantes de medicina}

\author{
Ubaldo Rodríguez-De Ávila1 (iD), Mónica Reyes-Rojas² ${ }^{(1)}$, Adalberto Campo-Arias ${ }^{3}$ (D), Carmen \\ Caballero-Domínguez ${ }^{4}$, Guillermo Ceballos-Ospino ${ }^{5}$ (D)
}

$\mathrm{D}$ esde hace varias décadas, las entrevistas en los procesos de selección de estudiantes para las escuelas de Medicina han sido repetidamente objeto de discusión. No obstante, se ha investigado muy poco sobre la utilidad de la entrevista clásica en la admisión de los futuros médicos ${ }^{1,2}$.

Un hallazgo que se puede tomar a favor de la entrevista lo presentaron Azman et al ${ }^{1}$; estos investigadores observaron que los estudiantes admitidos por entrevistas puntuaron más alto en rasgos de personalidad como extroversión, escrupulosidad, agradabilidad y franqueza y más bajo en neuroticismo que aquellos elegidos sin entrevista. Mientras que Rahbar et al ${ }^{2}$ observaron que las puntuaciones en la entrevista de admisión fueron pobres predictores del rendimiento en Anatomía, Bioquímica y Salud Comunitaria e, inclusive, los estudiantes con altas puntuaciones en la entrevista mostraron calificaciones más bajas en Fisiología.

Pese a la limitada evidencia que invite a una u otra recomendación, varias universidades de amplio y reconocido prestigio han abandonado esta estrategia no solo porque estiman que la entrevista aporta poca información relevante, sino también porque constituye un elemento de discriminación para estudiantes con características culturales diferenciales ${ }^{3,4}$. En consecuencia, la entrevista se convierte en un elemento que genera desigualdad y propicia la inequidad para los aspirantes en situación de desventaja ${ }^{3,5}$. Los estudiantes con mayores ingresos económicos o de grupos sociales con privilegios generalmente reciben calificaciones más altas y son admitidos ${ }^{3,6}$. Asimismo, Kim et al plantean que las entrevistas de admisión distan de evaluar o reconocer el perfil o rasgos de los aspirantes para lograr una formación médica óptima, es decir, de las características necesarias para las competencias requeridas y, adicionalmente, los criterios de selección son vagos e inespecíficos.

Además, se considera que la entrevista puede convertirse en un estresor para aquellos estudiantes no admitidos en un proceso anterior ${ }^{8}$. La entrevista no solo representa una presión considerable $y$, en algunos casos, conlleva agotamiento extremo, sino que también puede tener efectos negativos a largo plazo en los estilos de aprendizaje $e^{9}$

1.UniversidadeFederaldo Rio Grande do Norte.Natal,Brasil.Correo:rodriguez.ubaldo@gmail.com-http://orcid.org/0000-0001-5907-001X

2. Universidad del Magdalena. Santa Marta, Colombia. Correo: mreyesr@unimagdalena.edu.co - http://orcid.org/0000-0001-6077-7127

3. Universidad del Magdalena. Santa Marta, Colombia. Correo: acampo@unimagdalena.edu.co - http://orcid.org/0000-0003-2201-7404

4.Universidad del Magdalena. Santa Marta, Colombia.Correo: ccaballero@unimagdalena.edu.co - http://orcid.org/0000-0003-3730-2750

5. Universidad del Magdalena. Santa Marta, Colombia. Correo: guillermoceballos@gmail.com - http://orcid.org/0000-0002-1568-7058 
El proceso de selección de estudiantes de medicina y otras profesiones es un proceso complejo, sin que en la actualidad se cuente con una solución definitiva que siga principios de objetividad, justicia y equidad. La entrevista tradicional no es la mejor opción ${ }^{1,2}$. Sin duda, la utilización de un conjunto o batería de instrumentos o mini-entrevistas que evalúen diferentes habilidades, capacidades o destrezas puede facilitar una mejor selección de estudiantes; sin embargo, los estudios no son concluyentes ${ }^{1,7,10}$.

\section{DECLARACIÓN DE CONFLICTOS DE INTERESES}

Ninguno que declarar.

\section{REFERENCIAS BIBLIOGRÁFICAS}

1. Azman MAZ, Yaacob NA, Yusoff MSB, Noor SH. Comparative study on medical student personality traits between interview and noninterview admission method in University Sains Malaysia. Procedia. 2014; 116: 2281-5.

2. Rahbar MH, Vellani C, Sajan F, Zaidi AA, Akbarali L. Predictability of medical students' performance at the Aga Khan University from admission test scores, interview ratings and systems of education. Med Educ. 2001; 35: 374-80.

3. Curtis E, Wikaire E, Jiang Y, McMillan L, Loto R, Reid P. A tertiary approach to improving equity in health: quantitative analysis of the Māori and Pacific Admission Scheme (MAPAS) process, 2008-2012. Int J Equity Health. 2015; $14: 7$.
4. Kelly ME, Dowell J, Husbands A, Newell J, Siun OF, Kropmans T, et al. The fairness, predictive validity and acceptability of multiple mini interview in an internationally diverse student population-a mixed methods study. BMC Med Educ. 2014; 14: 267.

5. Zimdars A. Fairness and undergraduate admission: a qualitative exploration of admissions choices at the University of Oxford. Oxford Rev Educ. 2010; 36: 307-23.

6. Stevens M. Creating a class: college admissions and the education of elites: Boston: Harvard University Press; 2007.

7. Kim KJ, Nam KS, Kwon BS. The utility of multiple mini-interviews: experience of a medical school. Korean J Med Educ. 2017; $29: 7$.

8. Monteiro MA, Savalli C, Cúri M, Gorenstein $\mathrm{C}$, Andrade LH. Mood changes in the course of preparation for the Brazilian university admission exam-a longitudinal study. Rev Bras Psiquiatr. 2010; 32: 30-6. Doi: http://dx.doi. org/10.1590/S1516-44462010000100007

9. Seddigh R, Abdollahpour E, Azarnik S, Shariati $B$, Keshavarz-Akhlaghi AA. Participating in university entrance exam despite repeated failure: a qualitative study of participants' experiences. Int J Med Educ. 2016; 7: 345-53.

10. Knorr M, Schwibbe A, Ehrhardt M, Lackamp J, Zimmermann S, Hampe W. Validity evidence for the Hamburg multiple miniinterview. BMC Med Educ. 2018; 18: 106. 